\title{
Magnetic resonance imaging of the brain and spinal cord in cerebrotendinous xanthomatosis
}

\author{
Katherine S Bencze, Donald R Vande Polder, Leon D Prockop
}

\begin{abstract}
This reports a 40 year old man with cerebrotendinous xanthomatosis who had bilateral cataracts, enlarged Achilles tendons, progressive dementia, gait disturbance and peripheral neuropathy. Electroencephalography, electromyography, and magnetic resonance imaging (MRI) of the brain and spine were performed. Magnetic resonance imaging revealed cerebral, cerebellar and cervical cord atrophy and white matter involvement in the cerebrum and cerebellum correlating well with the clinical findings. To date there has been one previous report of MRI of the brain in cerebrotendinous xanthomatosis and none of the spinal cord.
\end{abstract}

Cerebrotendinous xanthomatosis (CTX) is a rare autosomal recessive disorder of bile acid metabolism. It results in abnormally high cholestanol concentration in plasma, cerebrospinal fluid (CSF) and tissues. ${ }^{1}$ Affected patients develop juvenile cataracts, progressive dementia, gait disturbance, peripheral neuropathies and tendon xanthomas. ${ }^{2}$

Early recognition is important because treatment with chenodeoxycholic acid can not only halt the progression of this potentially lethal condition but improve neurological function. ${ }^{34}$ Neurodiagnostic studies play an important role in diagnosing cerebrotendinous xanthomatosis and guiding treatment. This report correlates the magnetic resonance imaging (MRI) and electroencephalographic findings with the clinical presentation.

\footnotetext{
Department of Neurology, College of Medicine, University of South Florida,

Tampa, Florida, USA

K S Bencze

D R Vande Polder

L D Prockop

Correspondence to:

Katherine S Bencze MD Neurology Service, Veterans' Hospital,

13000 Bruce B Downs Blvd,

Tampa, Florida 33612, USA

Received 14 July 1989.

Accepted 6 September 1989
}

\section{Case report}

We report the case of a 40 year old man. His parents were second cousins and have no other children. No other family members suffered neurological disease. Early developmental milestones were normal following a normal vaginal delivery. He attended regular school classes until third grade when he transferred to special education classes which he attended through the tenth grade. At the age of nine years he developed bilateral cataracts. Enlarged Achilles tendons were first noted when he was 18 years. He performed manual labour with some difficulty until the age of 33 when the onset of progressive speech difficulty, lower extremity weakness and memory deficits prevented gainful employment. Except for a lifelong history of diarrhoea, he suffered no general medical problems.

Examination showed that he was thin, looked ill and had dysmorphic facial features, pectus excavatum, scoliosis and pes cavus. He was only oriented to person and did very poorly on short-term memory testing. His general knowledge was limited. There was decreased muscle bulk in the lower extremities with weakness. He showed mild dysmetria and decomposition of rapid successive hand movements and a broad-based ataxic gait. Deep tendon reflexes were brisk bilaterally with flexor plantar responses. He had decreased vibration sense in the lower extremities.

Laboratory tests showed serum electrolytes, liver function tests, complete cell count,

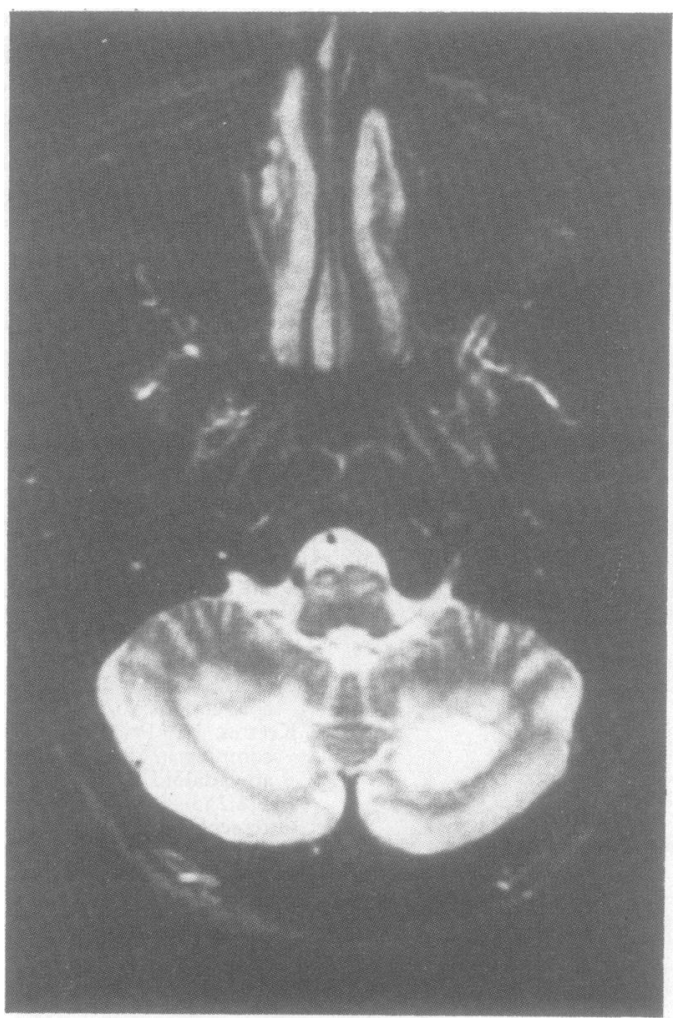

Figure 1 T2 weighted axial magnetic resonance image (TR2400, TE90, one excitation $256 \times 256$ matrix, $5 \mathrm{~mm}$ thick slides) axial view is shown at the level of the cerebellum: increased signal intensity in the cerebellar hemispheres (white matter) is seen bilaterally with prominent cerebellar folia. This is compatible with cerebellar white matter disease and atrophy. 
Figure 2 T2 weighted magnetic resonance image (TR2700, TE90 with $5 \mathrm{~mm}$ thick slides, $250 \times 250$ matrix, one excitation) of the cervical spine shows mild cervical cord atrophy.

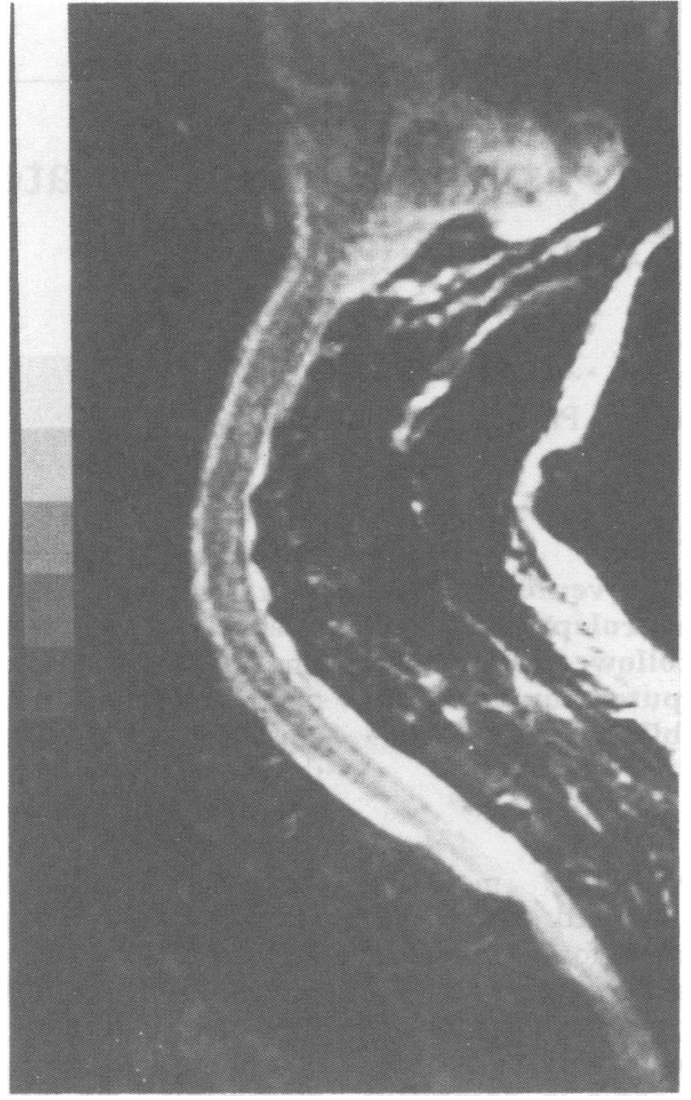

platelet count, and cholesterol level were normal. Serum cholestanol was $3.5 \mathrm{mg} / \mathrm{dl}$ (normal $<0.2 \mathrm{mg} / \mathrm{dl}$ ). Electromyography was consistent with axonal-demyelinating sensorymotor polyneuropathy. Electroencephalography showed diffuse bilateral slowing. MRI of the brain and spine showed marked cerebellar and moderate cerebral, cervical cord atrophy and abnormal signal in the cerebellar white matter (figs 1,2).

\section{Discussion}

CTX is a rare autosomal recessive disorder with low or normal serum cholesterol levels and high cholestanol levels with progressive neurological dysfunctions (dementia, ataxia, peripheral neuropathy), cataracts and coronary atherosclerosis. ${ }^{5}$ In 1968 Menkes, Schimschock and Swanson recognised that the brain tissue of these patients contained large amounts of cholestanol (the 5-dihydro-derivative of cholesterol). ${ }^{6}$

Pathological studies ${ }^{7}$ disclosed cerebral and cerebellar atrophy and extensive secondary demyelination due to sterol accumulation in the cerebellum, posterior and lateral columns of the spinal cord. Lipid storage was found in the cytoplasm of perivascular mononuclear cells.

The MRI findings of our patient support the clinical presentation. Diffuse cerebral atrophy accounts for the progressing dementia while white matter disease of the cerebellum correlates with ataxia and speech disturbance. The lower extremity weakness and diffusely brisk deep tendon reflexes may correlate with cervical cord involvement.

MRI is a more sensitive tool for detecting white matter disease in the central nervous system than computed tomography $;^{89}$ however, it cannot differentiate among different disease processes such as leukodystrophies, Binswanger's disease or multiple sclerosis. The presence of both brain and spinal cord demyelination narrows down the differential diagnosis to diseases that affect both, for example, multiple sclerosis and cerebrotendinous xanthomatosis.

MRI may become valuable in establishing the diagnosis of CTX early and monitoring treatment efficacy. With better understanding of the underlying biochemical abnormalities in CTX, treatment became possible and it encourages clinicians to establish the diagnosis early in the course of this disease. Hepatic bile synthesis is defective in CTX and it results in reduced cholic acid and chenodenoxycholic acid synthesis and continuous deposition of cholestanol as well as replacement of cholesterol with cholestanol in the central and peripheral nervous system. Chenodeoxycholic acid treatment has stabilised the disease and often improved neurological function. ${ }^{34}$

1 Berginer VM, Foster NL, Sadowsky M, Townsend JA, Siegel GJ, Salen G. Psychiatric disorders in patients with cerebrotendinous xanthomatosis. Am J Psychiatry 1988 145:354-7.

2 Kuritzky A, Berginer VM, Korczyn AD. Peripheral neuropathy in cerebrotendinous xanthomatosis. Neurology 1979;29:880-1.

3 Salen G, Berginer V, Shore V, Horak I, Horak E, Tint GS, Shefer S. Increased concentrations of cholestanol and apolipoprotein B in the cerebrospinal fluid of patients with polipoprotein B in the cerebrospinal fluid of patients with 316:1233-8.

4 Pedley TA, Emerson RG, Warner CL, Rowland LP, Salen $G$. Treatment of cerebrotendinous xanthomatosis with chenodeoxycholic acid. Ann Neurol 1985;18:517-8.

5 Salen G, Grundy SM. The metabolism of cholestanol, cholesterol and bile acids in cerebrotendinous xanthomatosis. J Clin Invest 1973;52:2822-35.

6 Menkes JH, Schimschock JR, Swanson PD. Cerebrotendinous xanthomatosis. The storage of cholestanol within the nervous system. Arch Neurol 1968;19:47-53.

7 Schimschock JR, Alvord EC, Swanson PD. Cerebrotendinous xanthomatosis. Clinical and pathological studies. Arch Neurol 1968;18:688-98.

8 Swanson PD, Cromwell LD. Magnetic resonance imaging in cerebrotendinous xanthomatosis. Neurology 1986;36: $124-6$.

9 Berginer VM, Berginer J, Salen G, Shefer S, Zimmermann $\mathrm{RD}$. Computed tomography in cerebrotendinous xanthomatosis. Neurology 1981;31:1463-5. 